\title{
Fast moving texture has opposite effects on the perceived speed of visible and occluded object trajectories
}

\author{
Luca Battaglini PhD ${ }^{\mathrm{a}, *}$, Giulio Contemori M.S. ${ }^{\mathrm{a}}$, Marcello Maniglia PhD ${ }^{\mathrm{b}, \mathrm{c}}$, Clara Casco Professor ${ }^{\mathrm{a}}$ \\ a Department of General Psychology, University of Padova, Via Venezia 8, 35131 Padova, Italy \\ b Centre de Recherche Cerveau \& Cognition (CerCo), Toulouse, France \\ c University of California, Riverside, Department of Psychology, Riverside, CA, USA
}

\section{A R T I C L E I N F O}

\section{Article history:}

Received 30 April 2016

Received in revised form 15 August 2016

Accepted 17 August 2016

Available online 29 August 2016

\section{Keywords:}

Motion

Invisible motion

Perceived speed

Texture

Surround motion

\begin{abstract}
A B S T R A C T
In a series of psychophysical experiments, we altered the perceived speed of a spot (target) using a grayscale texture moving in the same (iso-motion) or opposite (anti-motion) direction of the target. In Experiment 1, using a velocity discrimination task (2IFC), the target moved in front of the texture and was perceived faster with antimotion than iso-motion texture. The integration and segregation of motion signals in high-level motion areas may have accounted for the illusion. In Experiment 2, by asking observers to estimate the time-to-contact (TTC) with a bar indicating the end of the invisible trajectory, we showed that this illusory visible speed, due to anti- (iso-) texture, reduced (increased) the subsequent estimated duration of occluded target trajectory. However, in Experiment 3, when the target disappeared behind the iso-motion texture, the TTC was estimated shorter than anti- and static textures. In Experiment 4, using an interruption paradigm, we found negative Point of Subjective Equalities (PSEs) with iso-motion but not static texture, suggesting that iso-motion led to overestimation of the hidden speed. However, sensitivity to target speed differences, as assessed by JNDs and $d^{\prime}$ values was not affected. Results of Experiments 3 and 4 indicate that only the iso-texture affected the estimated target speed, but with opposite polarity compared to visible motion, suggesting a different origin of speed bias. Because our results show that visuospatial tracking was facilitated by the fast iso-motion, we conclude that motion of the occluded target was tracked by shifting visuospatial attention.
\end{abstract}

(C) 2016 Elsevier B.V. All rights reserved.

\section{Introduction}

Many objects continuously cross our visual field. At times, their trajectory becomes hidden by other objects for a brief period. In the 20th century, authors focussed on the perception of continuity (Burke, 1952; Michotte, 1946; Michotte, Thinès, \& Crabbé, 1964, 1991; Sampaio, 1943), referring to the period of occlusion in which the movement is "seen" by the observer's mind's eye as an "amodal phase" (Burke, 1952). As opposed to the modal phase, during which the moving object is visible, the motion of the object is invisible during the amodal phase. Previous studies have investigated the "amodal phase" with a prediction-motion task in which observers were required to judge when a moving object reappeared from behind an occluder; the time to contact (TTC) between the target and the further edge of the occluder was estimated (Benguigui \& Bennett, 2010; DeLucia \& Liddell, 1998; Makin \& Poliakoff, 2011; Makin, Poliakoff, Chen, \& Stewart, 2008; Makin, Poliakoff \& El-Deredy, 2009; Peterken, Brown, \& Bowman, 1991; Rosenbaum, 1975). Other studies used an interruption paradigm to investigate motion extrapolation, in which observers had

\footnotetext{
* Corresponding author.

E-mail address: luca.battaglini@unipd.it (L. Battaglini).
}

to report whether the target reappeared at the correct position (assuming a constant velocity during the occlusion) or not, instead of the time of contact (Cooper, 1989; DeLucia \& Liddell, 1998; Makin et al., 2008; Makin \& Poliakoff, 2011).

Several studies have investigated the similarities between visible and invisible motion. It has been shown that the relationship between the physical arrival time (actual TTC) and TTC (estimated) depends on different parameters, such as the target's speed, the size of the target (Battaglini, Campana, \& Casco, 2013; Sokolov \& Pavlova, 2003), the duration of the occlusion (Bennett, Baures, Hecht, \& Benguigui, 2010), and the presence of distractors (Horswill, Helman, Ardiles, \& Wann, 2005; Lyon \& Waag, 1995; Oberfeld \& Hecht, 2008; Reynolds, 1968). Moreover, several studies have shown that adapting the specific retinal zone in which the target will be occluded produces a shift in the TTC estimation; the estimated TTC was longer after motion adaptation in the same direction of the moving target, and shorter after adaptation in the opposite direction (Gilden, Blake, \& Hurst, 1995), which was also true for a rapid form of motion adaptation (Battaglini, Campana, Camilleri, \& Casco, 2015).

Here, we want to investigate the similarities between visible and invisible motion in a novel way. We ask how a moving texture can influence the perceived speed of a moving object that is either visible or 
invisible. Experiment 1 aims to replicate the compelling effect by which a moving visible target appears to move faster when a texture background moves in the opposite direction (Baker \& Graf, 2008; Baker \& Graf, 2010) and slower when a texture background moves in the same direction (Baker \& Graf, 2008; Loomis \& Nakayama, 1973). Both lower-level motion computation (Baker \& Graf, 2010) and motion processing in area MT may account for these illusory phenomena. Indeed, MT neurons are selective for the direction and speed of a moving stimulus, and play an important role in reducing noise, integrating different motion signals, and segregating target signals from noise (Born \& Bradley, 2005).

In the remaining three experiments, we ask whether similar contextual effects on motion processing occur during occlusion. This hypothesis is supported by the finding that visible motion and invisible (occluded) motion involve common neural mechanisms in the human brain (Olson, Gatenby, Leung, Skudlarski, \& Gore, 2004). However, a different scenario may occur, when considering that the estimated speed of the occluded target relies on (covert or overt) visuospatial attentive tracking (Makin \& Poliakoff, 2011), guided by memory of velocity (Battaglini et al., 2013). If memory of velocity is affected by the faster texture speed, then the speed of the invisible target may be overestimated when it moves in the same direction of the texture.

Experiments 2, 3 and 4 aim to investigate whether the speed illusion due to the texture is also present when motion is invisible and is reflected in the judgement of either the TTC values (prediction-motion task) or whether the target reappeared early or late (interruption paradigm).

\section{Experiment 1}

A moving target appears to move faster when textured background elements move in the opposite direction, and slower when they move in the same direction (Baker \& Graf, 2008; Baker \& Graf, 2010). However, there are exceptions; for example, Norman, Norman, Todd, and Lindsey (1996) found that the speed of a cloud of dots seen through a circular aperture (target) is seen as being slower when an annular surrounding region of moving dots was added, regardless of whether the direction of the dots was in the same or opposite direction (Norman et al., 1996). In this experiment, we then probe the role of a directionally moving texture on the perceived speed of a target moving in front of the texture. We asked participants to determine which of two targets presented in a two-interval forced-choice paradigm (2IFC) was moving faster. One target was the standard stimulus (SS) with fixed speed, and the other one was the comparison stimulus (CS), whose speed varied to different levels.

\subsection{Method}

\subsubsection{Participants}

Seven students from the University of Padova took part in this experiment. They were three males and four females aged between 23 and 26; all were right handed and had normal or corrected-to-normal vision. All of the participants in this and the following experiments were naive with respect to the purpose of the experiment and gave written informed consent according to the Declaration of Helsinki prior to their inclusion in the experiment. They all had normal or corrected-tonormal visual acuity.

\subsubsection{Apparatus}

The participants were seated in a dark room, $57 \mathrm{~cm}$ from the display screen. The viewing was binocular. Stimuli were generated with MATLAB and the Psychophysics Toolbox (Brainard, 1997; Pelli, 1997), and were displayed on a 19-in. CTX CRT Trinitron monitor with a refresh rate of $100 \mathrm{~Hz}$. We used a gamma-corrected lookup table (LUT) so that luminance was a linear function of the digital representation of the image. The screen resolution was $1024 \times 768$ pixels. Each pixel subtended $\sim 1.9^{\prime}$. The maximum luminance was $125 \mathrm{~cd} / \mathrm{m}^{2}$, and the minimum luminance was $0.9 \mathrm{~cd} / \mathrm{m}^{2}$. Luminance was measured using a Minolta LS-100 photometer.

\subsubsection{Stimulus}

The target consisted of a bright spot moving against a texture, either static or moving, with a constant position on the screen (Fig. 1). The texture was made of a random array of pixels with different values of grey (the pixels' colour varied between white and black with 256 levels of grey) and a mean luminance of $28 \mathrm{~cd} / \mathrm{m}^{2}$. Motion of the texture pixels in the same direction (iso-motion) and in the opposite direction (antimotion) with respect to the target motion was obtained by shifting the position of the pixels rightwards or leftwards within a rectangular window of $8 \times 3 \mathrm{deg}$ centred on the screen. The texture speed was $16 \mathrm{deg} / \mathrm{s}$. In the first frame, the bright spot target $(0.5 \mathrm{deg}$ in diameter, $125 \mathrm{~cd} / \mathrm{m}^{2}$ ) appeared abruptly on the texture, 4 deg to the left or right from the centre of the screen, with equal probabilities (to avoid the buildup of directional aftereffects). The spot was moved along a horizontal trajectory either to the left or to the right, with equal probability, randomly, towards the opposite side of the texture by shifting its position by a fixed amount from frame to frame. The spot's speed was either fixed at $3 \mathrm{deg} / \mathrm{s}$, for the standard stimulus (SS) or it varied from trial to trial in five levels for the comparison stimulus (CS): 2.8, 2.9, 3.02, 3.1, $3.2 \mathrm{deg} / \mathrm{s}$. The SS and CS always moved in the same direction (Fig. 1). The spot disappeared abruptly when it reached the end of the texture (after $8 \mathrm{deg}$ ). A red dot $\left(0.1 \mathrm{in}\right.$ diameter, $24 \mathrm{~cd} / \mathrm{m}^{2}$ ) placed $0.2 \mathrm{deg}$ above the centre of the texture was the fixation mark.

\subsubsection{Experimental procedure}

Participants were tested with a two-interval forced-choice (2IFC) velocity discrimination task in which they had to report whether the moving spot was faster in the first or second interval. In each trial, SS (the spot with fixed speed) and CS (the spot with variable speed) were randomly presented in sequence, separated by an interval of $500 \mathrm{~ms}$. The participants were instructed to maintain fixation on a red spot in the centre of the screen and to respond by pressing the appropriate key at the end of the trial. The next trial started $1000 \mathrm{~ms}$ after the response. No feedback was given. The experiment consisted of four blocks; one for each condition with 60 randomly presented trials (12 repetitions $\times 5$ speed levels). In the four blocks, the CS and SS moved in the same direction and trajectory in two different intervals, at speed that was either fixed (SS) or variable (CS). In the first condition, CSanti/SSiso, the interval containing the SS had the target and texture moving in the same direction, whereas in the other interval, the direction of the texture was reversed with respect to that of the CS. In the second condition CSiso/SSanti, CS had the same direction as the texture in one interval, whereas in the second interval, the direction of the texture was reversed with respect to that of the SS. In the other two conditions, the interval with SS had a static texture instead, whereas the interval with CS had a texture moving in either the same direction (CSiso/ SSstatic) or in the opposite direction of the target (CSanti/SSstatic).

\subsection{Results}

Fig. 2 represents the proportion of "CS is faster" responses as a function of $\Delta$ speed in deg/s. There were more "CS is faster" responses with CS moving in front of an anti-motion texture, regardless of whether the SS moved against an iso- or static texture. The proportions of CS seen as faster were analysed using an ANOVA with Condition and Speed level $(2.8,2.9,3,3.1,3.2 \mathrm{deg} / \mathrm{s})$ as factors. The GreenhouseGeisser correction for degrees of freedom was applied here and in the following ANOVAs, where appropriate, i.e. when the sphericity of the data was violated, as indicated by a significant Mauchly's test. The ANOVA revealed a significant effect of Speed level $\left(F_{(3,18)}=60.88\right.$, $\mathrm{p}<0.001, \eta_{\mathrm{p}}^{2}=0.91$ ), meaning (Fig. 2) that the CS was seen as being faster when it was actually faster and vice versa. Most importantly, 


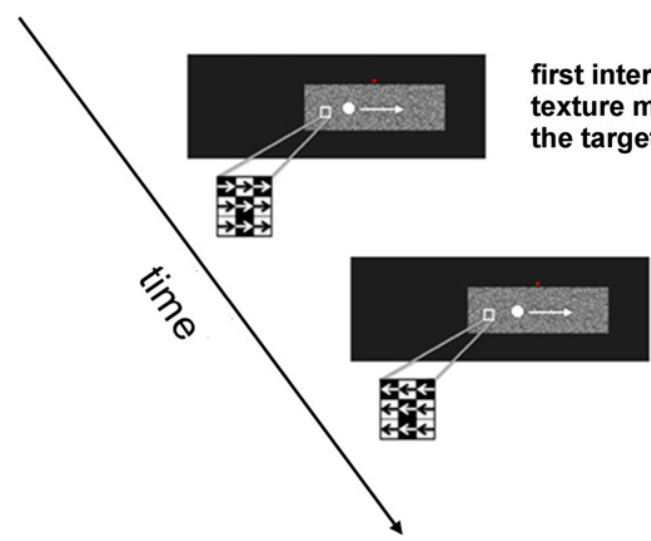

\section{irst interval}

exture moving in the same direction of

the target (iso-motion texture)

ISI $500 \mathrm{~ms}$

second interval

texture moving in the opposite direction

of the target (anti-motion texture)

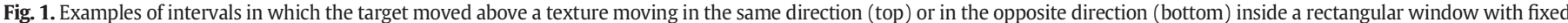

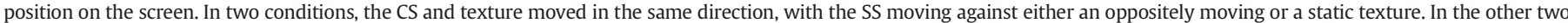
conditions, the SC and texture had opposite directions, whereas the SS had either the same direction as the target or was static.

the effect of Condition was significant $\left(\mathrm{F}_{(3,18)}=110.69, \mathrm{p}<0.001\right.$, $\eta_{p}^{2}=0.94$ ), whereas the interaction was not significant. Post-hoc comparisons (Bonferroni correction) showed that the proportion of "CS is faster" responses was significantly higher in CSanti/SSstatic than CSiso/SSstatic $(\mathrm{p}<0.001)$. Moreover, CSanti/SSiso was perceived as faster than CSiso/SSanti $(\mathrm{p}<0.001)$. There were no significant differences between CSanti/SSiso and CSanti/SSstatic $(p=0.99)$. Also, no differences were found between CSiso/SSanti and CSiso/SSstatic ( $\mathrm{p}=$ 0.81 ). One-sample t-tests revealed that when the CS and SS were equally fast, the proportions of "faster" responses were $>0.5$ in the two antimotion conditions (CSanti/SSiso, $\mathrm{p}=0.002$; CSanti/SSstatic, $\mathrm{p}=0.001$ ) and lower than 0.5 in the two iso-motion conditions (CSiso/SSanti, $\mathrm{p}=$ 0.012; CSiso/SSstatic, $\mathrm{p}=0.08$ ).

These results indicate that the anti-motion texture sped up the perceived speed of the CS, whereas the iso-motion texture slowed it down.

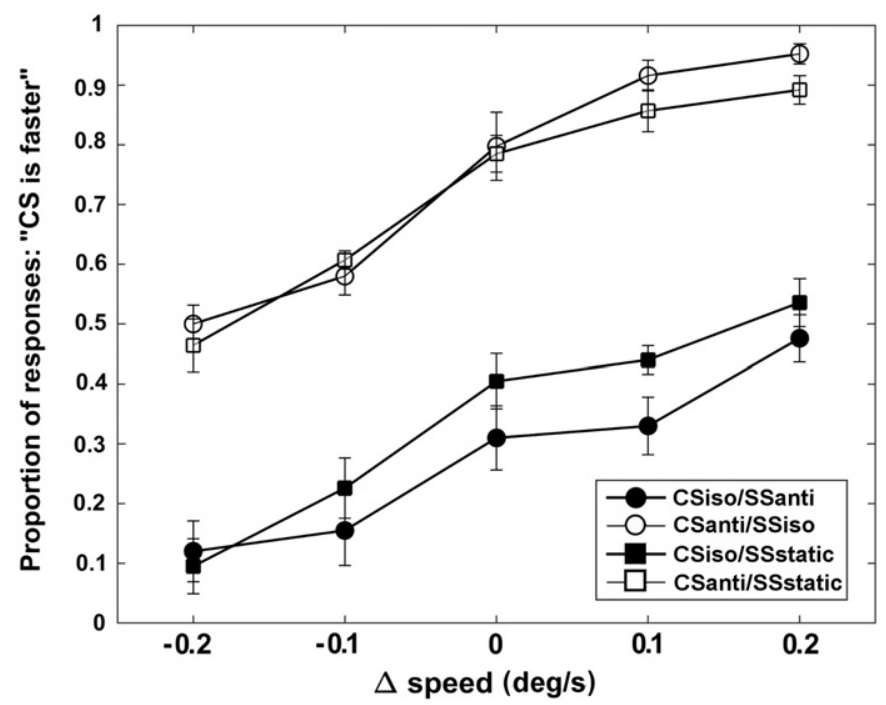

Fig. 2. The proportion of CS seen as faster is plotted as a function of $\Delta$ speed levels for the four conditions of background texture above which the SC moved. In the legend, the letters within brackets indicate whether the SC and the SS moved above a texture in iso(i) or anti-moving (a) textures. The filled circles indicate that the SC was moving above an iso-motion texture and the SS was moving above an anti-motion one. The empty circles indicate the opposite. The filled squares represent the condition in which the CS was moving above an iso-texture and the SS above a static one, while the empty squares represent the condition in which the CS was moving above an anti-texture and SS above a static one.

\section{Experiment 2}

The results of Experiment 1 showed that anti-motion texture speed up the visible target motion, while the iso-motion produced the opposite effect. In Experiment 2, we asked whether this speed bias would affect a subsequent estimation of the time to contact (TTC) of an occluded moving target in a prediction motion task. Therefore, in this experiment, the spot moved in front of an iso-, anti-, or static texture during the visible trajectory, and then disappeared behind an invisible occluder, and the participants had to estimate the time to contact between the occluded spot and a visible bar that indicated the end of the occlusion.

\subsection{Method}

\subsubsection{Participants}

Sixteen observers with normal or corrected-to-normal vision (eight females and eight males between 19 and 34 years of age) participated in this experiment.

\subsubsection{Apparatus and stimuli}

The apparatus was the same as in Experiment 1. The stimulus consisted of one single spot trajectory. The texture was made as in Experiment 1 ; when moving in either the same direction as or opposite direction to the target, its speed was $16 \mathrm{deg} / \mathrm{s}$ (as in Experiment 1). The spot (Fig. 5) appeared $10 \mathrm{deg}$ to the left or right from the centre of the screen, with equal probability, and moved in front of the texture $(8 \times 3 \mathrm{deg})$ along a 6 deg trajectory; then, it disappeared under an invisible occluder (same colour as the background) with the same size of the texture. A grey bar $\left(50 \mathrm{~cd} / \mathrm{m}^{2}\right)$ indicated the end of the invisible trajectory. Fig. 3 shows a schematic representation of the stimuli.

\subsubsection{Experimental procedure}

Participants were tested on a prediction-motion task with estimation of time-to-contact (TTC), i.e. the time between moving behind the occluder and reaching the bar (Fig. 3). Observers were instructed to maintain fixation on the red spot and to press the spacebar when they estimated the leading edge of the moving target reached a grey bar indicating the end of the invisible trajectory. No feedback was given. The experimental block consisted of 120 randomly presented trials resulting from 20 repetitions of each combination of speed ( $3 \mathrm{vs}$ $6 \mathrm{deg} / \mathrm{s}$ ) and texture motion (iso-, anti-, and static). The inter-trial interval was $1000 \mathrm{~ms}$. The participants were told that the target maintained constant speed and direction behind the occluder. 


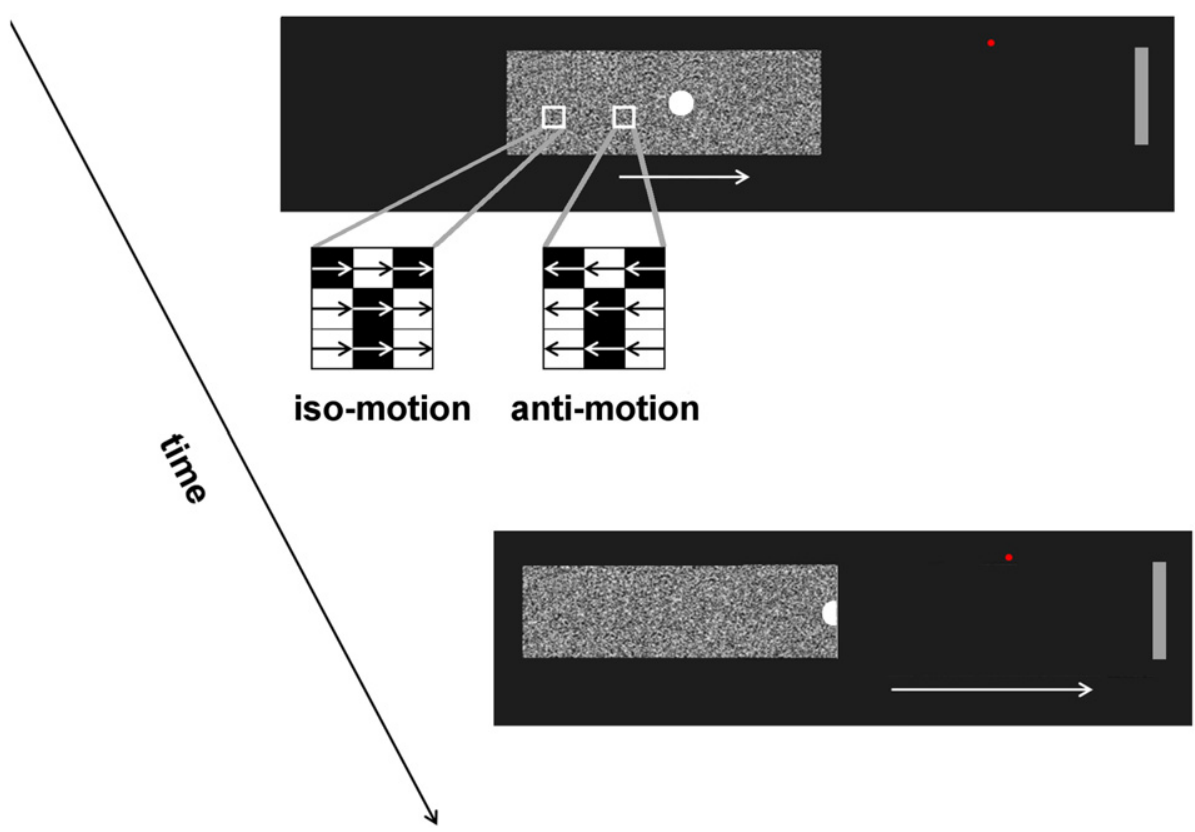

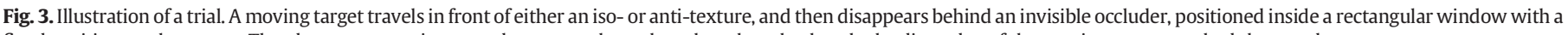
fixed position on the screen. The observers were instructed to press a key when they thought that the leading edge of the moving target reached the grey bar.

\subsection{Results}

Fig. 4 shows the TTCs and implied speed (as derived by the ratio between TTC and occluded trajectory length) obtained in the anti-, iso-, and static motion conditions. The TTCs were estimated as being shorter with the anti-texture than iso-texture, whereas the TTCs obtained with static textures are in between.

We analysed the mean estimated TTC of the invisible trajectory duration with a two-way repeated measures ANOVA, with Speed (low vs high), and Texture motion (iso-, anti-, and static) as the factors. The results revealed a significant effect of Speed $\left(\mathrm{F}_{(1,15)}=140.23, \mathrm{p}<0.001\right.$, $\left.\eta_{\mathrm{p}}^{2}=0.96\right)$, the mean estimated TTCs were faster with a high target speed (4.63 vs $2.65 \mathrm{~s}$ ). Most importantly, the effect of Texture motion was significant $\left(\mathrm{F}_{(1.285,19,272)}=12.579, \mathrm{p}=0.001, \eta_{\mathrm{p}}^{2}=0.46, \varepsilon=\right.$ $0,64)$. Bonferroni-corrected post-hoc t-tests indicated that the TTCs were estimated as being longer when the visible spot moved in front of an iso-texture compared to anti-motion $(p=0.002)$ and static textures $(\mathrm{p}=0.035)$. Moreover, the TTC was significantly shorter with anti-motion than static texture $(\mathrm{p}=0.036)$. The Speed $\times$ Texture motion interaction was significant $\left(\mathrm{F}_{(2,30)}=13.68, \mathrm{p}<0.001, \eta_{\mathrm{p}}^{2}=\right.$ 0.47). Bonferroni-corrected post-hoc t-tests revealed shorter TTCs with anti- than iso- $(\mathrm{p}=0.02)$ and static textures $(\mathrm{p}=0.034)$, but there was no difference between the static and iso-textures ( $\mathrm{p}=$ 0.73 ) at low speed. Instead, at high speed, the TTC was longer with an iso- compared to an anti-motion $(\mathrm{p}=0.001)$ and static $(\mathrm{p}=0.002)$ texture, but there was no difference between the static and anti-textures $(\mathrm{p}=0.46)$.

Thus, increasing (reducing) the perceived speed during the visible trajectory resulted in a shorter (longer) TTC.

\section{Experiment 3}

The results of Experiment 1 showed that a texture in anti-motion speeds up the visible motion of the target, whereas a texture in iso-motion slows it down. The effect was compelling, as reported in previous works (Baker \& Graf, 2008; Baker \& Graf, 2010). Experiment 2 showed that this speed bias influenced a subsequent TTC that was estimated as being shorter with the anti- compared to the static texture, and longer with the iso- compared to the anti- and static textures. Experiment 3 probed the role of texture motion presented over the occluder on the invisible target's motion. After its visible trajectory on the grey background, the disk disappeared behind an iso-motion, anti-motion, or static texture. Predictions of target motion were assessed using a TTC task (TTC with the further edge of the texture; see fig. 5).
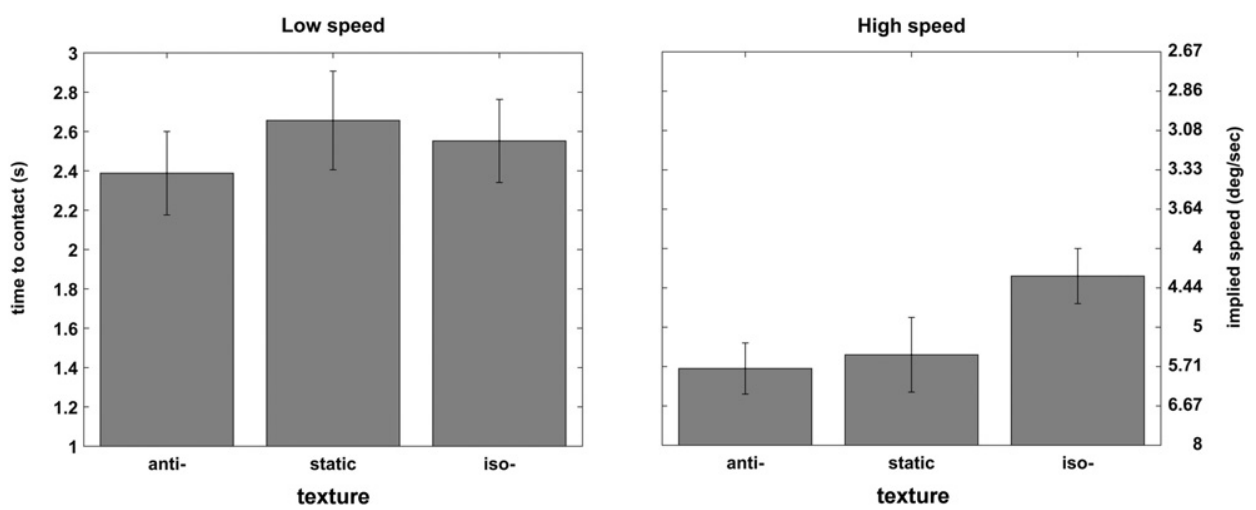

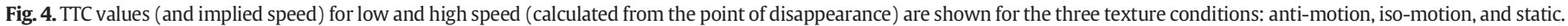




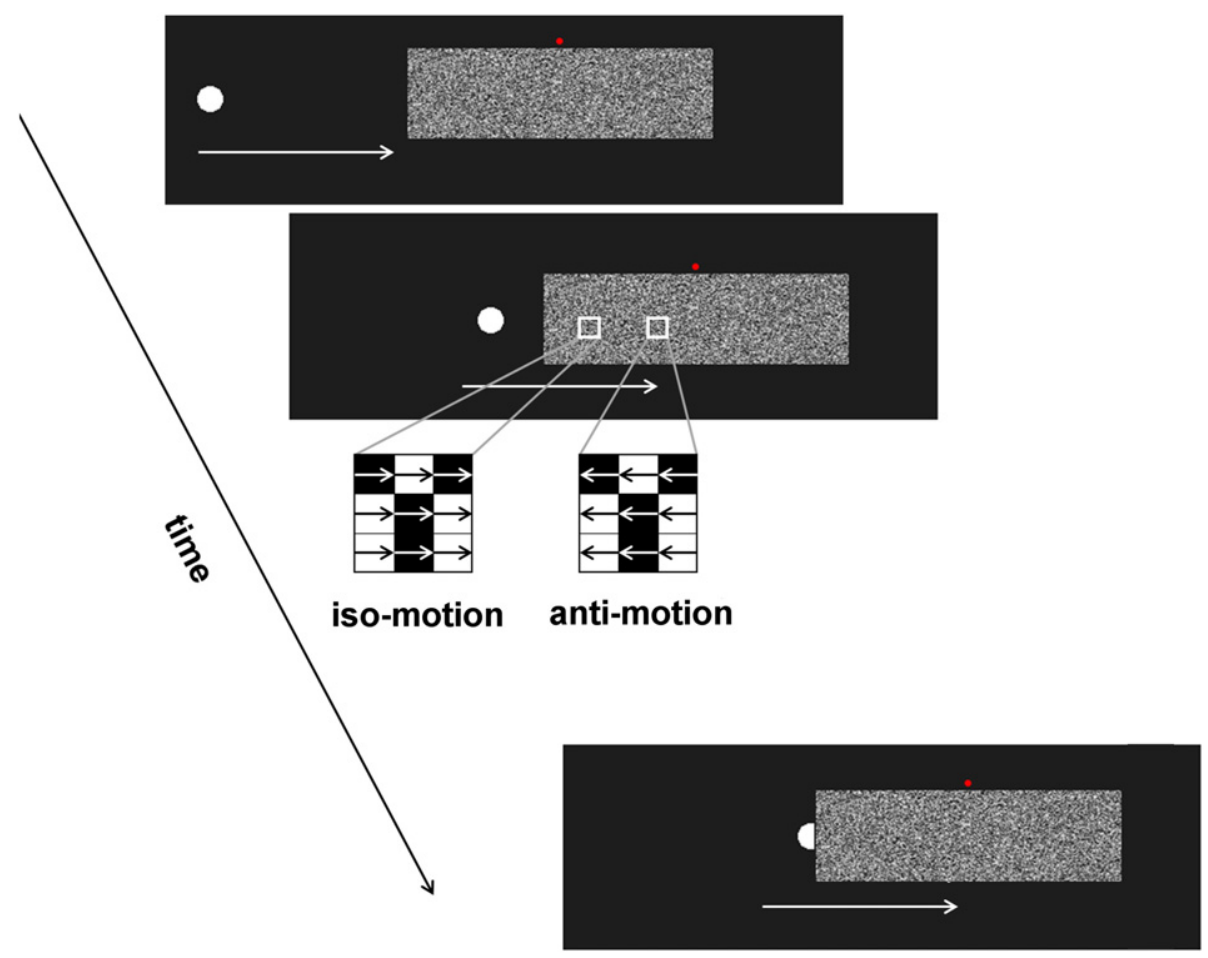

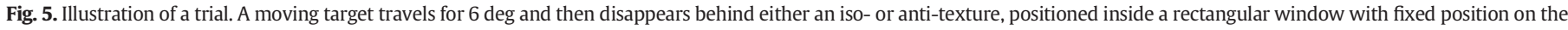
screen. The observers were instructed to press a key when they thought that the leading edge of the moving target reached the final edge of the texture.

We considered two hypotheses. The first was that visible and invisible motion would be similarly affected by the moving texture. Indeed, fMRI data indicate that visible motion and invisible (occluded) motion activate similar cortical regions in humans (Olson et al., 2004). Therefore, we expected shorter TTCs when spot and texture had opposite directions than when they had the same direction of motion. The second hypothesis stemmed from the suggestion that mechanism underlying prediction-motion task operates on the basis of memory of pre-occluded target speed (Battaglini et al., 2013; Makin \& Poliakoff, 2011). If memory of velocity is affected by texture speed visible during occlusion, then estimated speed of the invisible target may be biased towards texture speed.

\subsection{Method}

\subsubsection{Participants}

Sixteen observers with normal or corrected-to-normal vision (eight females and eight males aged between 21 and 35 years of age) participated in this experiment.

\subsubsection{Apparatus, stimuli and procedure}

The apparatus was the same as in Experiment 1. The texture size, motion type, fixation spot, extent of the visible motion trajectory, texture and target speeds were the same as in Experiment 2. In this experiment, the spot appeared $13 \mathrm{deg}$ to the left or the right from the centre of the screen, with equal probabilities (to avoid the build-up of directional aftereffects), and travelled with a horizontal trajectory towards the opposite side of the screen. Note that the target did not reappear from behind the texture. Fig. 5 shows a schematic representation of the stimuli. The procedure was the same as in Experiment 2.

\subsection{Results}

Fig. 6 shows the TTCs obtained in the anti-, iso-, and static motion conditions. TTCs were estimated as shorter with the iso-motion texture than the anti- and static textures. We analysed the mean TTC, i.e. the mean estimated TTC of the invisible trajectory duration, with a twoway repeated measures ANOVA, with Speed (low vs high), and Texture Motion (iso, anti and static) as the factors. The ANOVA showed a significant effect of Speed $\left(F_{(1,15)}=140.23, p<0.001, \eta_{p}^{2}=0.9\right)$, indicating that faster targets produced shorter TTC estimates. Most importantly, the effect of Texture motion was significant $\left(\mathrm{F}_{(2,30)}=6.98, \mathrm{p}=0.003\right.$, $\eta_{\mathrm{p}}^{2}=0.32$ ). Bonferroni-corrected post-hoc t-tests indicated that the TTC estimation with the iso texture is significantly shorter than the TTC estimation with the anti $(\mathrm{p}=0.012)$ and with the static texture $(\mathrm{p}=0.03)$. The interaction of Speed $\times$ Texture motion was not significant $\left(\mathrm{F}_{(2,30)}=1.23, \mathrm{p}=0.3, \eta_{\mathrm{p}}^{2}=0.08\right)$.

The results are not compatible with the hypothesis that visible motion and invisible motion are similarly affected by the moving texture. Instead, based on the assumption that the motion of occluded target is tracked by shifting visuospatial attention guided by memory of speed

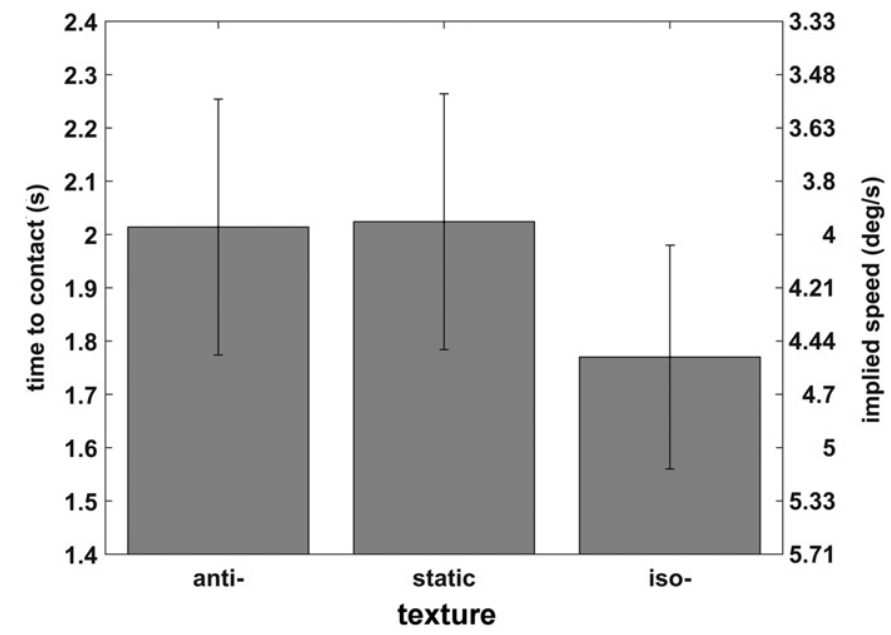

Fig. 6. TTC values averaged across speeds (calculated from the point of disappearance) are shown for the three texture conditions: anti-motion, iso-motion, and static. 
(Makin \& Poliakoff, 2011), our results support the hypothesis that memory of speed is biased towards texture speed, when texture and target have the same direction.

\section{Experiment 4}

In this experiment, we used an interruption paradigm (IP), in which the target reappeared either at the correct position or at a position either ahead or behind with respect to the correct one (DeLucia \& Liddell, 1998). The participants performed a discrimination task in which they had to report whether the target reappeared early or late, assuming it had travelled at a constant velocity across the occlusion interval. Note that the target reappeared on the background texture; this way the participants could not infer the point where the target would reappear (Fig. 5). Moreover, the occlusion duration and position of reappearance were unpredictable, so that there was no information about the relationship between velocity and occlusion duration. Only the target's velocity could be used to guide judgments. Based on the results of Experiment 3, we predicted that the target will be perceived as faster when occluded by the iso-motion texture compared to the static texture.

\subsection{Method}

\subsubsection{Participants}

Twenty-two volunteers, 10 males and 12 females, aged between 23 and 36, who were all right handed, took part in this experiment. They all had normal or corrected-to-normal vision.

\subsubsection{Apparatus and stimuli}

The apparatus was the same as in Experiment 1. The stimulus consisted of a single-spot trajectory. A schematic illustration of the stimulus is shown in Fig. 7. The target and fixation spot were as in Experiment 3 . The length of the invisible trajectory was 4,8 , or $12 \mathrm{deg}$, whereas the length of the background texture was always $18 \mathrm{deg}$. Note that the target reappeared in front of the texture. As in previous experiments three types of textures were used as in the previous experiment: iso-, anti-, and static, when in motion was $16 \mathrm{deg} / \mathrm{s}$. The target speed was always $3 \mathrm{deg} / \mathrm{s}$. The visible trajectory (6.3 deg) started $10.3 \mathrm{deg}$ from the centre (Fig. 7). Without altering the length of each invisible trajectory, a reappearance error of $\pm 0,150$, and 300 ms was added. After the reappearance, the target stopped and remained visible until the response.

\subsubsection{Procedure}

Participants were tested on an interruption paradigm in a motion extrapolation task (Cooper, 1989; DeLucia \& Liddel, 1998). After the spot trajectory ended, participants were instructed to press the appropriate key to indicate whether the target had reappeared earlier or later, even when the target reappeared in time. We used the psychophysical method of constant stimuli. Each block consisted of 300 randomly presented trials: 2 textures (static and moving) $\times 3$ invisible trajectory lengths $\times 5$ levels of reappearance errors $\times 10$ repetitions. The inter-trial interval was $1000 \mathrm{~ms}$ from key-press. No feedback was given. The participants performed two blocks, one devoted to comparing the static vs iso-textures and the other one devoted to comparing the static vs anti-textures. Each experimental block was preceded by 30 practice trials in which the target always reappeared in time (static texture $\times 3$ occluder lengths $\times 10$ repetitions). In each trial, the texture motion was either static or moving, in iso-motion in one block and antimotion in the other, with respect to the target's motion.

\subsection{Results}

The results of Experiment 4 are shown in Fig. 8 and Tables 1 and 2. Table 1 reports, proportion of response "late" obtained in the five reappearance errors conditions, for each invisible trajectory length and noise condition. Only for the short invisible trajectory length and iso-motion condition there was an effect of noise. Fig. 8 shows psychometric functions fitted to average proportion of responses "late" obtained as a function of reappearance error in the short invisible trajectory length condition. Iso-motion speeded up invisible motion whereas anti-motion had no effect. Sensitivity to reappearance errors was not affected by either noise, indicating that discrimination of invisible speed differences is unaffected by noise. Table 2 reports the $d^{\prime}$ values (sensitivity index) and criterion C obtained by averaging the accuracy obtained with positive and negative reappearance errors (hits) and incorrect responses (FA) obtained with no reappearance errors. Both the $d^{\prime}$ values and criterion $C$ were unaffected by whether the texture was static or moving.

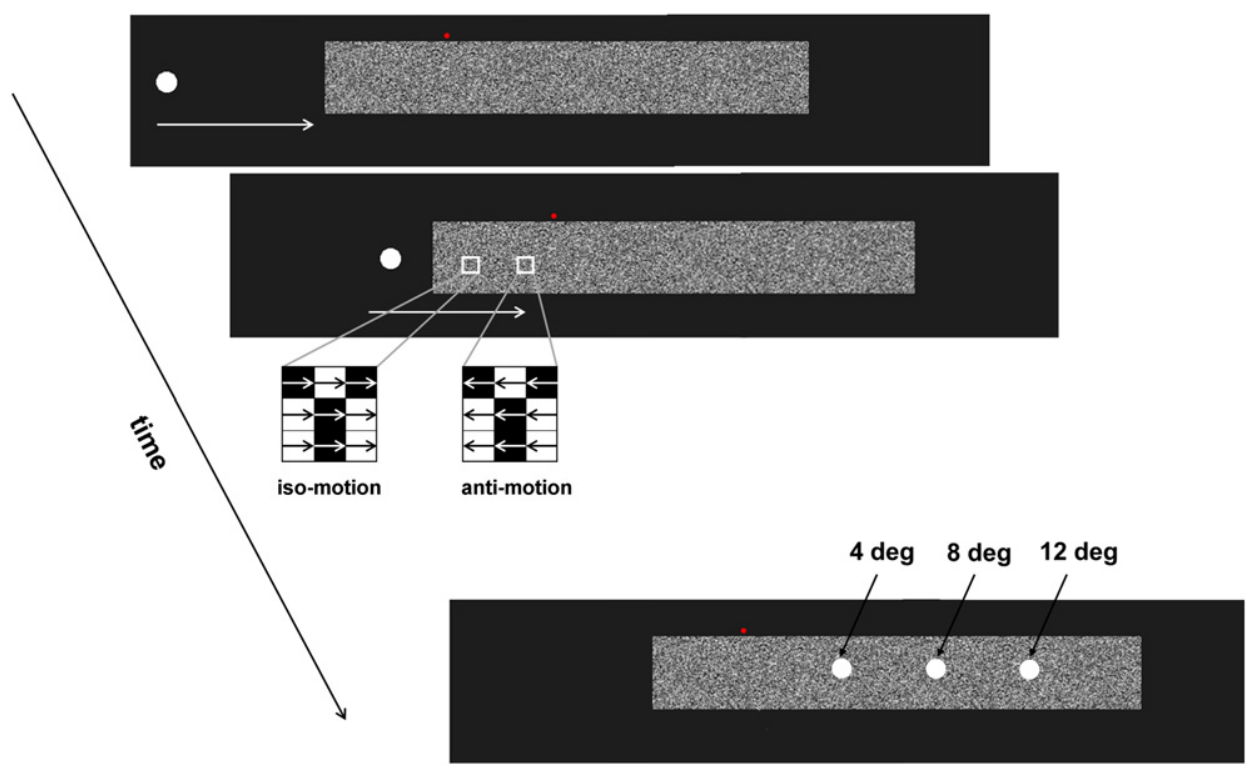

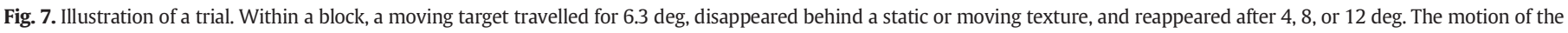

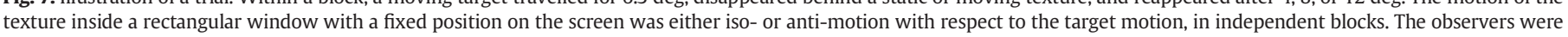
instructed to indicate whether the target reappeared late or early, assuming it maintained a constant speed behind the texture. 

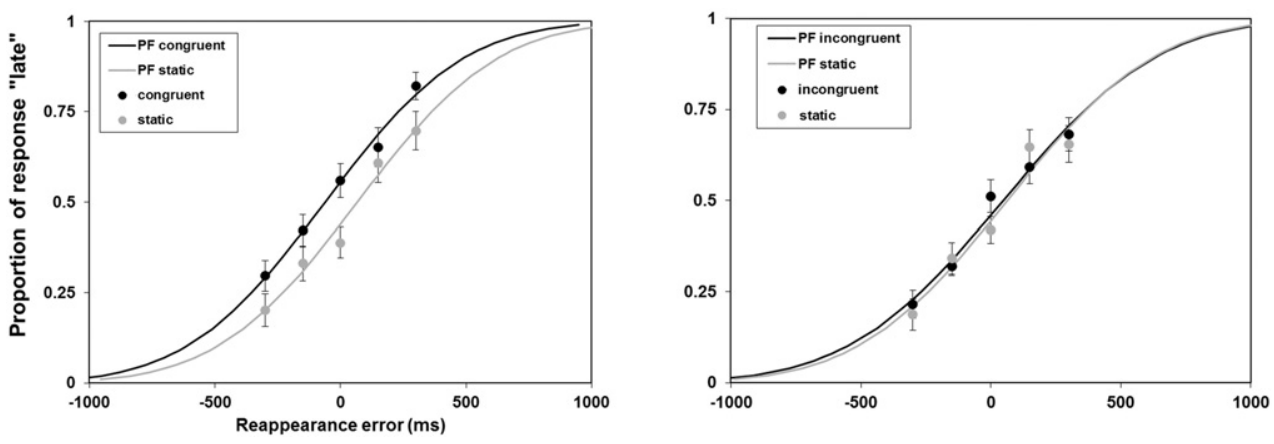

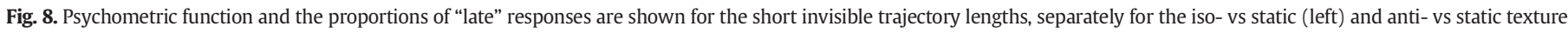
conditions (right).

In order to test the effect of noise on proportion of responses "late", we conducted a four-way repeated-measures ANOVA with texture motion direction (iso-motion vs anti-motion) texture type (nose vs static), invisible trajectory length $(4,8$, and $12 \mathrm{deg})$ and reappearance error $(-300,150,0,150,300)$ as factors. Significant effects were: texture direction $\left(F_{(1,21)}=4.54, p=0.045, \eta_{p}^{2}=0.18\right)$, invisible trajectory $\left(\mathrm{F}_{(2,42)}=5.3, \mathrm{p}=0.009, \eta_{\mathrm{p}}^{2}=0.2\right)$, reappearance error $\left(\mathrm{F}_{(1.621,34.041)}=53.6, \mathrm{p}=0.0001, \eta_{\mathrm{p}}^{2}=0.72\right)$, texture type $\left(\mathrm{F}_{(2,42)}=\right.$ $\left.4.2, \mathrm{p}=0.022, \eta_{\mathrm{p}}^{2}=0.17\right)$, texture direction $\times$ reappearance error $\left(\mathrm{F}_{(4,84)}=5.03, \mathrm{p}<0.001, \eta_{\mathrm{p}}^{2}=0.19\right)$, invisible trajectory $\times$ reappearance error $\left(\mathrm{F}_{(4.423,92.891)}=4.7, \mathrm{p}=0.001, \eta_{\mathrm{p}}^{2}=0.18\right)$ and, most importantly, texture direction $\times$ texture type $\times$ invisible trajectory $\left(\mathrm{F}_{(2,42)}=\right.$ $6.4, p=0.004, \eta_{p}^{2}=0.23$ ). Post hoc comparison (Bonferroni corrected $t$-test) revealed that in the iso-motion and short invisible trajectory condition, proportion of response "late" was higher with moving than static texture $(\mathrm{p}=0.01$; see Table 1$)$.

Speed overestimation (Point of Subjective Equality, or PSE) and sensitivity to speed differences (just noticeable difference, JND) were obtained by fitting Probit psychometric functions (Finney, 1971) to individual proportion of response "CS late" obtained as a function of reappearance error in the short invisible trajectory condition. Chi-square returned good fits for 18 participants. The JND, indicates the reappearance error value associated to 0.75 correct delay discrimination probability. The PSE indicates the reappearance error value at which the target was judged to reappear in time. A PSE $\leq$ or $>0$ indicates overestimation, no speed bias and underestimation of speed, respectively. Twoway ANOVAs were conducted to compare PSEs and JNDs, obtained with static and moving texture in the iso-motion and anti-motion condition. ANOVA on PSEs revealed a significant interaction of direction $\times$ texture type $\left(F_{(1,17)}=8.5, p=0.01, \eta_{p}^{2}=0.33\right)$ whereas the main factors did not resulted significant: motion direction $\left(\mathrm{F}_{(1,17)}=2.6, \mathrm{p}=0.12, \eta_{\mathrm{p}}^{2}=\right.$ $0.13)$, texture type $\left(F_{(1,17)}=1.9, p=0.19, \eta_{p}^{2}=0.1\right)$. Post-hoc comparison (Bonferroni corrected $t$-test) revealed a significant difference between static and moving texture in the iso-motion condition ( $\mathrm{p}=$ $0.002)$; in addition the PSE obtained with moving texture $\left(t_{(17)}=3.2\right.$ $\mathrm{p}=0.005)$ but not in the static texture $\left(\mathrm{t}_{(17)}=1.48 \mathrm{p}=0.16\right)$ was lower than 0 . In the anti-motion condition there was no difference in the PSE obtained with moving and static texture $(\mathrm{p}=0.79)$. The ANOVA comparing JNDs did not show any significant effect: texture direction $\left(\mathrm{F}_{(1,17)}=0.22, \mathrm{p}=0.65, \eta_{\mathrm{p}}^{2}=0.13\right)$, texture type $\left(\mathrm{F}_{(1,17)}=\right.$ $\left.0.35, \mathrm{p}=0.56, \eta_{\mathrm{p}}^{2}=0.02\right)$, texture direction $\times$ texture type $\left(\mathrm{F}_{(1,17)}=0.12, \mathrm{p}=0.73, \eta_{\mathrm{p}}^{2}=0.007\right)$.

With the aim of assessing whether the PSE effect was associated to a more liberal or more conservative response criterion we checked for differences in either sensitivity $\left(d^{\prime}\right)$ or response criterion (C) obtained from hits (obtained by excluding trials where the target reappeared in time and averaging correct responses "early" and "late" for 300 and 150 ms reappearance errors) and FA (referring from the difference from chance of the proportion "late" obtained in the no-delay condition). Two four-way repeated measures ANOVA were conducted, with congruency (iso vs anti), texture (moving vs static), invisible trajectory length $(4,8,12 \mathrm{deg})$, and the average of positive and negative delays ( $150 \mathrm{vs} 300 \mathrm{~ms}$ ) as factors. The ANOVA on $d^{\prime}$ only revealed a significant effect of delay $\left(F_{(1,21)}=12.7, p=0.002, \eta_{p}^{2}=0.38\right)$ and a significant interaction of delay $\times$ invisible trajectory $\left(\mathrm{F}_{(2,42)}=3.5, \mathrm{p}=0.04\right.$, $\eta_{\mathrm{p}}^{2}=0.14$ ), indicating (with Bonferroni correction) an effect of delay with short ( $p=0.001)$ and long invisible trajectory $(p=0.005)$. The ANOVA on criterion $C$ only revealed a significant effect of reappearance error $\left(F_{(1,21)}=21.8, p=0.0001, \eta_{p}^{2}=0.51\right)$.

\section{Discussion}

Experiment 1 showed that a spot in anti-motion to the texture motion was perceived as being faster than when it moved against an iso- or static texture. Moreover, when the observers compared two stimuli of equal speed, the one in anti-motion was perceived as being faster. Additionally, the increased perceived speed of the visible target moving in front of a texture moving in the opposite direction reduced the subsequent estimated TTC (Experiment 2), and the decrement of the target's perceived speed due to a texture moving in the same direction lengthened the estimated TTC. However, when the textures became the occluder (Experiment 3), we found an opposite pattern of results, but only with the iso-texture: the estimated TTC was shorter when the spot moved behind an iso-texture than an anti- or static texture, as if

Table 1

This table reports, for each invisible trajectory length and noise condition, response "late" obtained in the five reappearance errors conditions.

\begin{tabular}{|c|c|c|c|c|c|c|c|c|c|c|c|c|c|c|c|}
\hline & \multicolumn{15}{|c|}{ Invisible trajectory length } \\
\hline & \multicolumn{5}{|l|}{ Short } & \multicolumn{5}{|c|}{ Medium } & \multicolumn{5}{|l|}{ Long } \\
\hline & \multicolumn{5}{|c|}{ Reappearance errors } & \multicolumn{5}{|c|}{ Reappearance errors } & \multicolumn{5}{|c|}{ Reappearance errors } \\
\hline & -300 & -150 & 0 & 150 & 300 & -300 & -150 & 0 & 150 & 300 & -300 & -150 & 0 & 150 & 300 \\
\hline Iso & 0.30 & 0.40 & 0,56 & 0,65 & 0.77 & 0.38 & 0.40 & 0.50 & 0.56 & 0.58 & 0.29 & 0.33 & 0.34 & 0.41 & 0.48 \\
\hline Static & 0.19 & 0.37 & 0.40 & 0.59 & 0.70 & 0.24 & 0.38 & 0.45 & 0.58 & 0.62 & 0.24 & 0.34 & 0.44 & 0.47 & 0.53 \\
\hline Anti & 0.22 & 0.32 & 0.46 & 0.59 & 0.67 & 0.22 & 0.27 & 0.40 & 0.53 & 0.60 & 0.22 & 0.27 & 0.41 & 0.46 & 0.55 \\
\hline Static & 0.20 & 0.37 & 0.40 & 0.60 & 0.62 & 0.24 & 0.38 & 0.49 & 0.61 & 0.61 & 0.24 & 0.29 & 0.45 & 0.45 & 0.53 \\
\hline
\end{tabular}


Table 2

Sensitivity, $d^{\prime}$, and criterion, $C$, were derived according to signal detection theory from hits, obtained by averaging "early" and "late" correct reappearance errors and false alarms (FA), referring to incorrect responses obtained with 0 delay. The table report $d^{\prime}$ values and Cs were obtained with iso-motion and anti-motion textures (and static texture, respectively) separately for the different occluder lengths and reappearance errors.

\begin{tabular}{|c|c|c|c|c|c|c|c|c|c|c|c|c|}
\hline & \multicolumn{6}{|c|}{ Reappearance error $150 \mathrm{~ms}$} & \multicolumn{6}{|c|}{ Reappearance error $300 \mathrm{~ms}$} \\
\hline & \multicolumn{2}{|c|}{ Short } & \multicolumn{2}{|c|}{ Medium } & \multicolumn{2}{|l|}{ Long } & \multicolumn{2}{|l|}{ Short } & \multicolumn{2}{|c|}{ Medium } & \multicolumn{2}{|l|}{ Long } \\
\hline & $d^{\prime}$ & C & $d^{\prime}$ & C & $d^{\prime}$ & C & $d^{\prime}$ & C & $d^{\prime}$ & $\mathrm{C}$ & $d^{\prime}$ & C \\
\hline Iso & 1.22 & 0.27 & 1.32 & 0.48 & 1.01 & 0.43 & 1.55 & 0.22 & 1.38 & 0.45 & 1.2 & 0.34 \\
\hline Static & 1.30 & 0.22 & 1.37 & 0.46 & 1.13 & 0.41 & 1.65 & 0.09 & 1.44 & 0.28 & 1.32 & 0.3 \\
\hline Anti & 1.51 & 0.5 & 1.47 & 0.48 & 1.07 & 0.34 & 1.79 & 0.29 & 1.63 & 0.37 & 1.27 & 0.22 \\
\hline Static & 1.45 & 0.35 & 1.5 & 0.47 & 1.29 & 0.5 & 1.68 & 0.29 & 1.49 & 0.32 & 1.46 & 0.38 \\
\hline
\end{tabular}

the iso-texture sped up the occluded target. Experiment 4 showed that the participants only reported that the target reappeared late more often with a short iso-texture compared to a control condition (static texture), indicating that they tended to see the occluded target as moving faster with iso- compared to static texture. This illusory speed bias was not associated with a difference in sensitivity or response criterion.

A possible neural account for the speed effect in Experiment 1 is the response of motion detectors with centre-surround receptive fields in motion processing area MT (Born, Groh, Zhao, \& Lukasewycz, 2000; Tadin, Lappin, Gilroy, \& Blake, 2003). Indeed, it was shown that MT neurons stopped to respond to a moving target when surrounding motion in the same direction as the target was added (Born, Groh, Zhao, \& Lukasewycz, 2000), whereas their response to the preferred motion direction increased when the surrounding motion was in the opposite direction (Eifuku \& Wurtz, 1998). However, different explanations may be suggested. Baker and Graf (2010) suggested that direction-tuned suppressive and facilitatory interactions in the primary visual cortex (V1) may account for the speed illusion. Considering that our texture was larger than the classical moving surrounding motion used in previous works, long-range interactions in the near periphery may have contributed to modulating the strength of the speed illusion (Casco, Battaglini, Bossi, Porracin, \& Pavan, 2015). An interesting result is that speed overestimation during visible motion reduced TTCs, whereas speed underestimation increased TTCs. These results confirm the previous results that visible illusory speed affects invisible motion (Battaglini et al., 2013) and support the suggestion that illusory speed is retained in memory during "invisible motion".

We interpret the effect of iso-texture directionally on invisible (amodal) motion, as found in Experiment 3, as a confirmation of Makin and Poliakoff's (2011) model, stating that the occluded target's spatial position is updated thanks to the stored velocity memory that guide the shift of visuospatial attention. Indeed, shorter TTCs are expected when the target and texture have the same direction, assuming that memory of speed is affected by texture speed when in iso-motion. However, anti-motion did not increase the TTC, indicating that interference between visible texture speed and memory of target speed occurs when they have the same direction. Oberfeld, Hecht, and Landwehr (2011) obtained a similar asymmetry (but with opposite polarity) in a TTC estimation with approaching motion objects. They found that a contracting spiral caused an overestimation of TTC compared to a static one, but an expanding spiral did not result in a relative underestimation (Oberfeld et al., 2011).

The paradigm of Experiment 4 rules out the possibility of spurious effects in occluded motion judgments, due to timing mechanisms such as counting or changing the update of the internal clock (see Coull, Cheng, \& Meck, 2011, for a review); indeed, when we used an interruption paradigm, the participants could not calculate a priori the duration of the occlusion because they did not know where the target will reappear. Therefore, a timing strategy was not viable (DeLucia \& Liddell, 1998), and the observers could not use cues other than target velocity. The results of Experiment 4 confirm those obtained from the TTC task used in Experiment 3. Indeed, when the target reappeared in time, the participants responded "late" more often with the short iso-texture than with the short static texture. This means that they judged occluded target motion as being faster than it really was. As in Experiment 3, with anti-motion, we did not find speed underestimation or effects of texture on accuracy, indicating that amodal motion is affected by real motion vectors of the same but not opposite direction.

The speed overestimation with iso-motion was lost at medium (8 deg) and long (Cooper, 1989) occluder lengths, probably because the working memory system was saturated, thus impeding the conservation of object speed and its speed illusion. The difficulty of keeping the object speed behind the occluder may also explain the result that the participants were more accurate with short occluders compared to with long occluders when the target reappeared early or late. Moreover, the difficulty of keeping the object speed behind the occluder may have also slowed down the visuospatial update of the object, thus increasing the probability of "early" responses, even when the target reappeared in time or late.

\section{References}

Baker, D. H., \& Graf, E. W. (2008). Equivalence of physical and perceived speed in binocular rivalry. Journal of Vision, 8(4), 26.

Baker, D. H., \& Graf, E. W. (2010). Contextual effects in speed perception may occur at an early stage of processing. Vision Research, 50(2), 193-201.

Battaglini, L., Campana, G., \& Casco, C. (2013). Illusory speed is retained in memory during invisible motion. i-Perception, 4(3), 180-191.

Battaglini, L., Campana, G., Camilleri, R., \& Casco, C. (2015). Probing the involvement of the earliest levels of cortical processing in motion extrapolation with rapid forms of visual motion priming and adaptation. Attention, Perception, \& Psychophysics, 77(2), 603-612.

Benguigui, N., \& Bennett, S. J. (2010). Eye movements influence estimation of time-tocontact in prediction motion. Experimental Brain Research, 202, 327-339.

Bennett, S. J., Baures, R., Hecht, H., \& Benguigui, N. (2010). Eye movements influence estimation of time-to-contact in prediction motion. Experimental Brain Research, 206(4), 399-407.

Born, R. T., \& Bradley, D. C. (2005). Structure and function of visual area MT. Annual Review of Neuroscience, 28, 157-189.

Born, R. T., Groh, J. M., Zhao, R., \& Lukasewycz, S. J. (2000). Segregation of object and background motion in visual area MT: Effects of microstimulation on eye movements. Neuron, 26(3), 725-734.

Brainard, D. H. (1997). The psychophysics toolbox. Spatial Vision, 10(4), 433-436.

Burke, L. (1952). On the tunnel effect. Quarterly Journal of Experimental Psychology, 4(3), 121-138.

Casco, C., Battaglini, L., Bossi, M., Porracin, E., \& Pavan, A. (2015). Suppressive effects on motion discrimination induced by transient flankers are reduced by perceptual learning. Journal of Vision, 15(8), 25

Cooper, L. A. (1989). Mental models of the structure of visual objects. In B. E. Shepp, \& S. Ballesteros (Eds.), Object perception: Structure and process (pp. 91-119). Hillsdale, NJ: Erlbaum.

Coull, J. T., Cheng, R. K., \& Meck, W. H. (2011). Neuroanatomical and neurochemical substrates of timing. Neuropsychopharmacology, 36(1), 3-25.

DeLucia, P. R., \& Liddell, G. W. (1998). Cognitive motion extrapolation and cognitive clocking in prediction motion tasks. Journal of Experimental Psychology: Human Perception and Performance, 24(3), 901

Eifuku, S., \& Wurtz, R. H. (1998). Response to motion in extrastriate area MSTI: Centersurround interactions. Journal of Neurophysiology, 80(1), 282-296.

Finney, D. J. (1971). Probit analysis (3rd edition ). Cambridge University Press.

Gilden, D., Blake, R., \& Hurst, G. (1995). Neural adaptation of imaginary visual motion. Cognitive Psychology, 28(1), 1-16.

Horswill, M. S., Helman, S., Ardiles, P., \& Wann, J. P. (2005). Motorcycle accident risk could be inflated by a time to arrival illusion. Optometry and Vision Science, 82(8), 740-746.

Loomis, J. M., \& Nakayama, K. (1973). A velocity analogue of brightness contrast. Perception, 2(4), 425-428.

Lyon, D. R., \& Waag, W. L. (1995). Time course of visual extrapolation accuracy. Acta Psychologica, 89(3), 239-260.

Makin, A. D., \& Poliakoff, E. (2011). Do common systems control eye movements and motion extrapolation? The Quarterly Journal of Experimental Psychology, 64(7), 1327-1343.

Makin, A. D., Poliakoff, E., Chen, J., \& Stewart, A. J. (2008). The effect of previously viewed velocities on motion extrapolation. Vision Research, 48(18), 1884-1893.

Makin, A. D., Poliakoff, E., \& El-Deredy, W. (2009). Tracking visible and occluded targets: Changes in event related potentials during motion extrapolation. Neuropsychologia, 47(4), 1128-1137.

Michotte, A. (1946). The perception of causality. trans. TR Miles and E. Miles London: Methuen.

Michotte, A., Thinès, G., \& Crabbé, G. (1964). Les complements amodaux des structures perceptives. Institut de psychologie de l'Université de Louvain.

Michotte, A., Thinès, G., \& Crabbé, G. (1991). Amodal completion of perceptual structures. Michotte's experimental phenomenology of perception (pp. 140-167). 
Norman, H. F., Norman, J. F., Todd, J. T., \& Lindsey, D. T. (1996). Spatial interactions in perceived speed. Perception, 25(7), 815-830.

Oberfeld, D., \& Hecht, H. (2008). Effects of a moving distractor object on time-to-contact judgments. Journal of Experimental Psychology: Human Perception and Performance, 34(3), 605.

Oberfeld, D., Hecht, H., \& Landwehr, K. (2011). Effects of task-irrelevant texture motion on time-to-contact judgments. Attention, Perception, \& Psychophysics, 73(2), 581-596.

Olson, I. R., Gatenby, J. C., Leung, H. C., Skudlarski, P., \& Gore, J. C. (2004). Neuronal representation of occluded objects in the human brain. Neuropsychologia, 42(1), 95-104.

Pelli, D. G. (1997). The VideoToolbox software for visual psychophysics: Transforming numbers into movies. Spatial Vision, 10(4), 437-442.

Peterken, C., Brown, B., \& Bowman, K. (1991). Predicting the future position of a moving target. Perception, 20(1), 5-16.
Reynolds, H. N., Jr. (1968). Temporal estimation in the perception of occluded motion. Perceptual and Motor Skills, 26(2), 407-416.

Rosenbaum, D. A. (1975). Perception and extrapolation of velocity and acceleration. Journal of Experimental Psychology: Human Perception and Performance, 1(4), 395.

Sampaio, A. C. (1943). La translation des objets comme facteur de leur permanence phénoménale. (Warny).

Sokolov, A., \& Pavlova, M. (2003). Timing accuracy in motion extrapolation: Reverse effects of target size and visible extent of motion at low and high speeds. Perception, 32(6), 699-706.

Tadin, D., Lappin, J. S., Gilroy, L. A., \& Blake, R. (2003). Perceptual consequences of centresurround antagonism in visual motion processing. Nature, 424(6946), 312-315. 